\title{
Comparison of clinical characteristics of conventional and nickel free metal brackets before and after recycling
}

\author{
Rabinder Kaur ${ }^{1, *}$, Piush Kumar ${ }^{2}$, Shalaj Bhatnagar ${ }^{3}$, Payal Sharma ${ }^{4}$ \\ ${ }^{1}$ Senior Lecturer, ${ }^{2}$ Professor, ${ }^{4}$ Professor and HOD, Dept. of Orthodontics, I.T.S. Centre for Dental Studies \& Research, \\ Ghaziabad, Uttar Pradesh, India ${ }^{3}$ Private Practitioner, Uttar Pradesh, India
}

*Corresponding Author:

Email: drrabinderkaur@gmail.com

\begin{abstract}
Objective: To compare the clinical characteristics of conventional and Nickel-free metal brackets before and after recycling. Materials and Method: Total of 25 Nickel-free (Co-Cr alloy) premolar brackets MBT 0.022" slot and equal number of 17-4 Stainless Steel premolar brackets MBT 0.022" slot were used in the study. For evaluation of slot dimension, brackets were viewed at 40X magnification under Stereomicroscope. Acrylic jig was made by stabilising 0.019X0.025" Stainless Steel on which test bracket was placed and evaluated for frictional resistance by Universal Testing Machine (UTM). An occluso-gingival load via UTM was applied on bracket-adhesive interface for testing shear bond strength.

Results: Paired t- test values for slot dimension showed significant differences between the groups before recycling on both Face and Base. When comparison was made within the groups, there was significant difference in 17-4 Stainless Steel brackets before and after recycling on both Face and Base. For frictional resistance, paired t test showed significant difference within Nickel-free brackets before and after recycling. For shear bond strength, paired t- test showed significant difference within the 17-4 Stainless Steel brackets before and after recycling.

Conclusion: Shear bond strength of recycled brackets was greater than as-received brackets for both 17-4 Stainless Steel and Nickel-free brackets. The slot dimensions of as-received brackets exhibited a wider dimension at face than at the base. On recycling, Nickel-free brackets showed a significant increase in frictional values.
\end{abstract}

Keywords: Frictional resistance, Stainless steel brackets, Slot dimension, Shear bond strength.

\section{Introduction}

One of the most common problems associated with bonding is the accidental "debonding" of the brackets before the treatment is completed. ${ }^{1}$ Also sometimes prior to the completion of orthodontic treatment, intentional debonding is required when brackets are not ideally positioned which often necessitates repositioning. ${ }^{2}$ The easier way is to use a new bracket every time but it may not be possible in case of anteriors where we may need to have a separate inventory for debonded brackets. The most commonly used solution is to recycle the debonded brackets.

Thus, bracket recycling has emerged concurrently with the direct bonding techniques. The clinical implications of recycling brackets is the alteration which occurs in the bracket morphology. ${ }^{3}$ These alterations may lead to alteration in the arch wire bracket friction, the bracket slot dimension and the bond strength. These changes will influence the retraction mechanics and thus the final orthodontic finish. $^{4}$

The effects of recycling depends on the method of debonding, type of reconditioning process used and material from which bracket is constructed, whether the bracket is milled or cast and the design of the bracket base. ${ }^{5}$ The various reconditioning procedures used in orthodontics include: burning technique, electropolishing, microetching, grinding, Big Jane method, Buchman method, non acid solution, ultrasonic cleaning, fine or rough diamond bur, silica coating. ${ }^{1,6}$
Sandblasting with $60 \mu \mathrm{m}$ alumina for 3 seconds at a distance of $10 \mathrm{~cm}$ has been shown by Scanning Electron Microscope examination to produce the best micro roughened surface to allow effective mechanical bonding. ${ }^{5}$

One of the major problems with conventional brackets is nickel sensitivity. To overcome this problem manufacturers have developed Nickel free (cobalt chromium) brackets for clinical usage. Cobalt chromium (Co-Cr) brackets are marketed as biocompatible alternatives to the stainless steel brackets because they contain little or no nickel, with the manufacturers claiming added benefit of lower frictional levels than the industry standard in low friction-stainless steel brackets. ${ }^{7,8}$ There is little in the orthodontic literature comparing the effect of recycling of Nickel free brackets and stainless steel brackets.

The null hypothesis for the present study was that there is no difference in the mechanical properties of as-received and recycled brackets manufactured by two different manufacturers.

\section{Materials and Method}

This study was approved by the ethical committee of the institution.

A total of 25 Nickel-free (Co-Cr alloy) premolar brackets MBT- 0.022" slot and equal number of 17-4 Stainless steel premolar brackets MBT- 0.022" slot were used for this study (Table 1). The experimental procedure involved the evaluation of slot dimension of 
the brackets followed by evaluation of the frictional resistance of the brackets. The brackets were then bonded to extracted premolar teeth for evaluation of shear bond strength. The debonded brackets were then recycled following which all the mechanical properties were re-evaluated. The same procedure was followed for both groups 1 and 2.

Table 1: Distribution of samples in various groups and subgroups

\begin{tabular}{|c|c|c|c|}
\hline Group & Brackets Used & Subgroup a & Subgroup b \\
\hline $\begin{array}{c}\text { Group 1 } \\
\mathrm{n}=25\end{array}$ & $\begin{array}{c}\text { 17-4 Stainless steel brackets } \\
\text { (MBT- 0.022 slot). }\end{array}$ & $\begin{array}{c}\text { As-received brackets } \\
(\mathrm{n}=25)\end{array}$ & $\begin{array}{c}\text { Recycled brackets } \\
(\mathrm{n}=25)\end{array}$ \\
\hline $\begin{array}{c}\text { Group 2 } \\
\mathrm{n}=25\end{array}$ & $\begin{array}{c}\text { Nickel-free (Co-Cr Alloy) } \\
\text { brackets (MBT-0.022 slot). }\end{array}$ & $\begin{array}{c}\text { As-received brackets } \\
(\mathrm{n}=25)\end{array}$ & $\begin{array}{c}\text { Recycled brackets } \\
(\mathrm{n}=25)\end{array}$ \\
\hline
\end{tabular}

a) Evaluation of slot dimension: The brackets were viewed under a Stereomicroscope at $40 \mathrm{X}$ magnification (Fig 1). The position of the brackets was stabilized using a putty so as to provide a clear view of the slot walls from the side of the bracket. Each bracket was scanned and captured individually in the stereomicroscope on both the mesial and the distal sides to produce a digital image. The images were exported and calibrated with Dewinger Biowizard 4.3 (Dewinter Optical Inc. India). The software used was accurate upto a least count of 1 micron or upto 5 decimals in inches. The slot dimension was measured both at face and at the base. The measurements were rounded off at the $4^{\text {th }}$ decimal. Each bracket was measured thrice at both the base and at face end of slot and a mean value was obtained. The mean of the left and right side was taken for the purpose of evaluation.

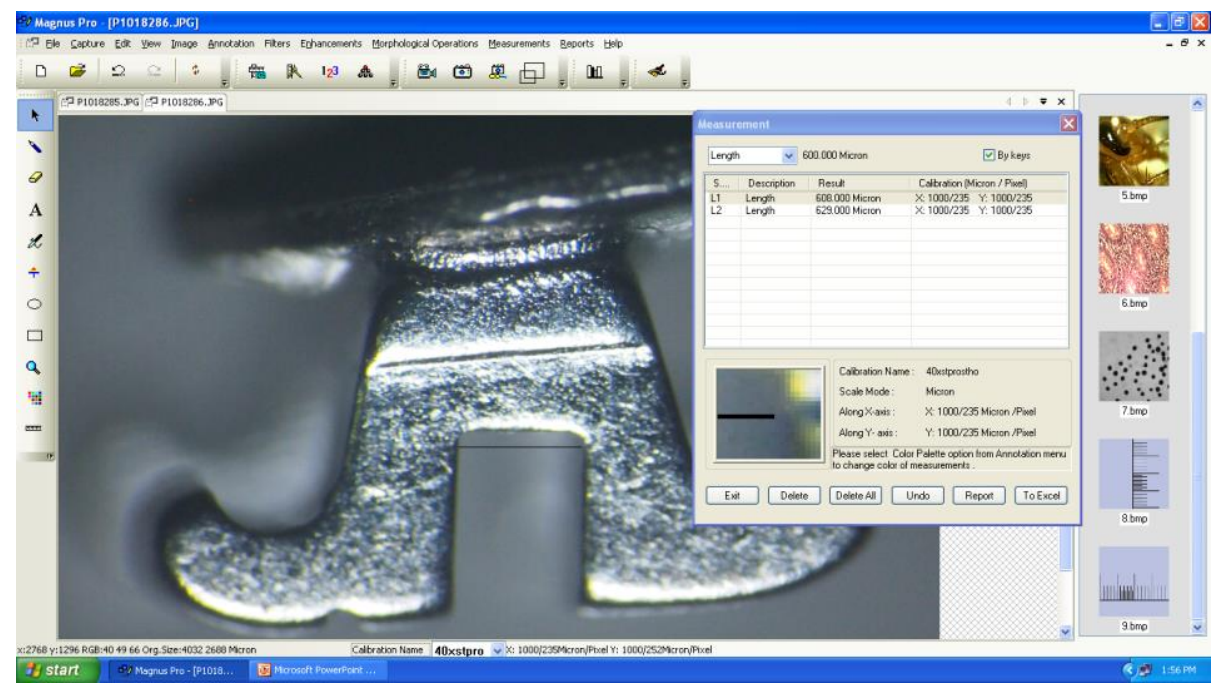

Fig. 1: Screenshot showing the horizontal line at base of slot taken from software of stereomicroscope

b) Evaluation of frictional resistance: The frictional resistance was tested on a Universal Testing Machine at room temperature in the dry state. An experimental set up was made by stabilizing a 0.019X0.025" stainless steel wire in an acrylic jig so that the wire was parallel to the long axis of the universal testing machine (Fig. 2). The testing bracket was attached to the stabilized arch wire on the jig with an elastic module placed with the help of Elastic shooter. The same person placed the elastomeric module immediately before each test to avoid force decay. Before testing, the bracket and the arch wires were cleaned with $95 \%$ alcohol. The test brackets were pulled through the jig at a speed of $5 \mathrm{~mm} /$ minute for one minute. The force registered in Newtons was converted to grams as follows:

1 Newton $=101.9 \mathrm{gm}$

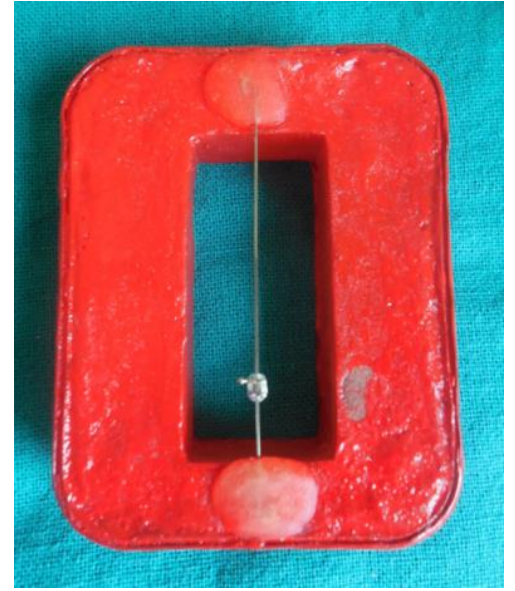

Fig. 2: Acrylic jig with mounted 0.019X0.025 inch stainless steel wire with Nickel-Lite bracket 
c) Evaluation of shear bond strength: The teeth were embedded in acrylic resin using moulds. Prior to bonding the orthodontic attachment to the tooth surface, it was prepared with pumice and rubber prophylactic cups for 10 seconds using a slow speed handpiece, washed with water spray and dried with oil free air spray.

Bonding procedure: The enamel surface was treated with Transbond self etching primer according to manufacturer's instructions and then cured for 10 seconds. Small layer of adhesive Transbond XT was applied to bracket base following which the bracket was positioned on the pretreated enamel surface, pressed with a $250 \mathrm{gms}$ force and excess adhesive was removed. Brackets were cured with a LED light curing unit having $600 \mathrm{~mW}$ intensity for 10 seconds from both the proximal sides.

Thermocycling procedure: After 24 hours, the mounted teeth were thermocycled between $5^{\circ} \mathrm{C}$ and $55^{\circ} \mathrm{C}$ for 500 cycles. The exposure to each bath was 20 seconds and the transfer time between the two baths was 5-10 seconds.

For bond strength evaluation the samples were tested on a Universal Testing Machine. An occlusogingival load was applied to the bracket, producing a shear force at the bracket tooth-interface. A blade was placed at the bracket base enamel interface and moved at a crosshead speed of $0.5 \mathrm{~mm} /$ minute until rupture of the bracket tooth union.

The bracket base area for 17-4 Stainless steel brackets (MBT- 0.022" slot) was $9.61 \mathrm{~mm}^{2}$ as per information provided by the manufacturer. ${ }^{9}$

The bracket base surface area for Nickel-free (Co-Cr Alloy) brackets (MBT- 0.022" slot) was 11.55 $\mathrm{mm}^{2}$ as per information provided by the manufacturer. ${ }^{9}$

\section{Recycling Procedure}

All the samples were recycled by a portable Sandblasting unit. Aluminium oxide $(50 \mu \mathrm{m})$ particles were used for sandblasting for 15 seconds from a distance of $3 \mathrm{~mm}$. Brackets were agitated in acetone for 20 seconds to ensure removal of any debris. After the brackets were recycled the slot dimension, frictional resistance and shear bond strength were tested again in a manner similar to the one explained above to obtain the post recycled values. All the values obtained were recorded and then evaluated statistically.

\section{Statistical Analysis}

The data obtained was subjected to statistical analysis using SPSS 16.0.0. Paired t test was used for comparison of the slot dimension, friction and shear bond strength before and after the recycling procedure.

\section{Results}

Paired t- test values for slot dimension showed significant differences between the groups before recycling on both Face and Base i.e ( $\mathrm{p}=.017$ and .001) respectively (Table 2).

When correlation was made within the groups, there was significant difference in 17-4 stainless steel brackets before and after recycling on both Face and Base ( $\mathrm{p}=.011$ and .013$)$ respectively (Table2).

For frictional resistance, paired $\mathrm{t}$ test showed significant difference within Nickel-free brackets before and after recycling ( $\mathrm{p}=.001)$ (Table 3 ).

For shear bond strength, paired $t$ test showed significant difference within the 17-4 stainless steel brackets before and after recycling $(\mathrm{p}=.007$ and .008$)$ respectively (Table 4).

Table 2: Mean and standard deviation for slot dimension of as received and recycled brackets

\begin{tabular}{|c|c|c|c|c|c|}
\hline & \multicolumn{2}{|c|}{ Before recycling } & \multicolumn{2}{|c|}{ After recycling } & \multirow{2}{*}{$\begin{array}{c}\begin{array}{c}\text { Paired } t \text { test } \\
\text { between various } \\
\text { subgroups }\end{array} \\
\text { p value }\end{array}$} \\
\hline & $\begin{array}{c}1 \mathrm{a}(17-4 \\
\text { SS })\end{array}$ & $\begin{array}{c}2 \mathrm{a}(\mathrm{Co}-\mathrm{Cr} \\
\text { alloy) }\end{array}$ & $\begin{array}{c}1 \mathrm{~b}(17-4 \\
\mathrm{SS})\end{array}$ & $\begin{array}{c}2 \mathrm{~b}(\mathrm{Co}-\mathrm{Cr} \\
\text { alloy) }\end{array}$ & \\
\hline Face & $\begin{array}{l}.0234 \pm \\
.0011\end{array}$ & $\begin{array}{l}.0208 \pm \\
.0052\end{array}$ & $\begin{array}{l}.0217 \pm \\
.0027\end{array}$ & $\begin{array}{l}.0228 \pm \\
.0045\end{array}$ & $\begin{array}{l}1 \mathrm{a} \text { vs } 1 \mathrm{~b}=.011^{* *} \\
1 \mathrm{a} \text { vs } 2 \mathrm{a}=.017^{* *} \\
2 \mathrm{a} \text { vs } 2 \mathrm{~b}=.175^{*} \\
1 \mathrm{~b} \text { vs } 2 \mathrm{~b}=.255^{*}\end{array}$ \\
\hline Base & $\begin{array}{l}.0230 \pm \\
.0007 .\end{array}$ & $\begin{array}{l}.0198 \pm \\
.0044\end{array}$ & $\begin{array}{l}.0214 \pm \\
.0028\end{array}$ & $\begin{array}{l}.0209 \pm \\
.0043\end{array}$ & $\begin{array}{c}1 \mathrm{a} \text { vs } 1 \mathrm{~b}=.013^{* *} \\
1 \mathrm{a} \text { vs } 2 \mathrm{a}=.001^{* * *} \\
2 \mathrm{a} \text { vs } 2 \mathrm{~b}=.295^{*} \\
1 \mathrm{~b} \text { vs } 2 \mathrm{~b}=.596^{*}\end{array}$ \\
\hline $\begin{array}{l}\text { Paired t } \\
\text { test face vs } \\
\text { base }\end{array}$ & $.042 * *$ & $.321 *$ & $.009 * * *$ & $.000 * * * *$ & \\
\hline
\end{tabular}

*p $>0.05$, not significant; $* * p<0.05$, significant; $* * * p<0.01$, very significant;

$* * * * \mathrm{p}<0.0001$, highly significant. 
Table 3: Mean and standard deviation for frictional resistance of as received and recycled brackets

\begin{tabular}{|l|c|c|c|}
\hline & Group 1(17-4 SS) & Group 2(Co-Cr alloy) & p value \\
\hline $\begin{array}{l}\text { Before recycling } \\
\text { Subgroup a }\end{array}$ & $246.31 \pm 64.13$ & $22.67 \pm 54.12$ & $1 \mathrm{a}$ vs $2 \mathrm{a}=.195^{*}$ \\
\hline $\begin{array}{l}\text { After recycling } \\
\text { Subgroup b }\end{array}$ & $274.48 \pm 58.76$ & $286.50 \pm 55.47$ & $1 \mathrm{~b}$ vs $2 \mathrm{~b}=.525^{*}$ \\
\hline p value & $1 \mathrm{a}$ vs $1 \mathrm{~b}=.167^{*}$ & $2 \mathrm{a}$ vs $2 \mathrm{~b}=.001^{* * *}$ & \\
\hline
\end{tabular}

$* \mathrm{p}>0.05$, not significant; $* * \mathrm{p}<0.05$, significant; $* * * \mathrm{p}<0.01$, very significant; $* * * * \mathrm{p}<0.0001$, highly significant.

Table 4: Mean and standard deviation for shear bond strength of as received and recycled brackets

\begin{tabular}{|l|c|c|c|}
\hline & Group 1(17-4 SS) & Group 2(Co-Cr alloy) & p value \\
\hline $\begin{array}{l}\text { Before recycling } \\
\text { Subgroup a }\end{array}$ & $7.76 \pm 3.97$ & $8.44 \pm 4.13$ & $1 \mathrm{a}$ vs $2 \mathrm{a}=.556^{*}$ \\
\hline $\begin{array}{l}\text { After recycling } \\
\text { Subgroup b }\end{array}$ & $11.70 \pm 6.02$ & $12.23 \pm 4.84$ & $1 \mathrm{~b}$ vs $2 \mathrm{~b}=.738^{*}$ \\
\hline $\mathrm{p}$ value & $1 \mathrm{a}$ vs $1 \mathrm{~b}=.007 * * *$ & $2 \mathrm{a}$ vs $2 \mathrm{~b}=.008^{* * *}$ & \\
& & & \\
\hline
\end{tabular}

$* \mathrm{p}>0.05$, not significant; $* * \mathrm{p}<0.05$, significant; $* * * \mathrm{p}<0.01$, very significant; $* * * * \mathrm{p}<0.0001$, highly significant.

\section{Discussion}

The various elements that should be considered when rebonding a bracket include: reconditioning of the enamel surfaces, the use of new/recycled bracket, the bonding system to be used and the bracket recycling method used.

As there is lack of consensus on the clinical characteristics of as-received and recycled brackets, so the aim of this study was to evaluate the clinical characteristics of 17-4 stainless steel brackets and Nickel-free (Co-Cr alloy) brackets and compare the differences between as-received and recycled brackets.

Over the years various materials have been used for manufacture of orthodontic brackets. Nickel sensitivity has been a paramount reason for manufacturers to develop brackets made of more biocompatible materials. Nickel hypersensitivity in the patients is estimated to be approximately $10 \%{ }^{7}$ Though stainless steel brackets with approximately $6-8 \%$ nickel content is generally considered to be safe, increased awareness of Nickel sensitivity and toxicity has led to the development of alternative systems. Nickel-free brackets are marketed as biocompatible alternatives to the stainless steel brackets because they contain little or no nickel, with the manufacturers claiming added benefit of lower frictional levels as compared to stainless steel brackets. ${ }^{7}$

The major advantage of recycling is the economic saving. Other advantages include a smoother, more corrosion resistant bracket after electropolishing. The disadvantages include reduction in bracket quality, loss of identification marks, lack of sterility and increased risk of cross infection. ${ }^{8}$ Some of the recycling techniques are- burning technique, electropolishing, microetching, grinding, Big Jane method, Buchman method, non acid solution, ultrasonic cleaning, fine or rough diamond bur, silica coating. ${ }^{8}$ It was reported that sandblasting alone produced insignificant effect on bond strength as compared to as-received brackets. ${ }^{6,8-10}$ As sandblasting is viable, time saving and convenient method of recycling so, sandblasting with 50 microns aluminium oxide was opted as a method of recycling brackets in this study.

It has been stated that the internal bracket slot dimension should be accurate upto $1 \mathrm{mil}(0.001 ") .{ }^{11}$ This gives a leeway of bracket slot size of 0.021 " to 0.023 ". Also the acceptable variation as given by Deutsches Institüt für Normung (DIN 13971-2) is a $0.04 \mathrm{~mm}$ $(0.00157 ")$ variation from the normal. ${ }^{12}$

In the present study, none of the brackets exhibited a 0.022 inch slot either at the base or at the face. The slot dimensions were generally greater at the face as compared to the base. The 17-4 stainless steel brackets exhibited values greater than 0.022 inch whereas the Nickel-free brackets had slot dimension less than 0.022 inch both at face and base. The slot dimensions of 17-4 stainless steel brackets conformed to the DIN standards. But in case of Nickel-free brackets the slot dimension at the base was less than that is acceptable by DIN standards. There was no major difference in the slot dimensions at the mesial and distal sides within both the groups. These findings are similar to studies in which 24 brackets from 8 manufacturers and found three bracket slots smaller and 20 others larger than the dimensions stated by their manufacturers. ${ }^{13}$ A previous study results showed that 17-4 stainless steel and Nickel free brackets showed slots with heights larger than those specified by the manufacturer. ${ }^{14}$

Previous study have also reported that the slot dimensions at face was generally more than at the base which is similar to the results obtained in the present study. ${ }^{15}$ In the recycled groups our study found that the 
slot dimension at face was slightly less than the nonrecycled groups. In 1989 it was reported that there may be a slot closing produced due to debonding procedure. ${ }^{16}$ On comparing the new and recycled brackets we found that in case of 17-4 stainless steel brackets there was a significant difference between the as-received and recycled slot dimensions both at face and base. This difference was present both at distal and mesial sides. In case of Nickel-free brackets no significant difference in the slot dimension between asreceived and recycled brackets both at mesial and distal sides were found.

Study done in 2005 had evaluated various brackets produced by MIM. ${ }^{17}$ They studied the hardness of various brackets and found that Nickel-free alloy brackets are generally harder than the ferrous alloy brackets. This may explain the greater distortion in the slot dimension that was achieved in 17-4 stainless steel brackets as compared to Nickel-free brackets.

Another interesting observation in the present study was that although there was a significant difference between the slot dimensions of as-received 17-4 stainless steel and Nickel-free brackets both at the base and face, after recycling this difference became insignificant. These findings indicate that for the purpose of recycling of the brackets it is probably better to use a Nickel-free alloy rather than a 17-4 stainless steel brackets. In the present study although the asreceived Nickel-free brackets showed a lower mean value as compared to as-received 17-4 stainless steel brackets: the difference was not statistically significant. This is contrary to the results obtained by Nair et al who showed significant difference in frictional forces between as-received Nickel-free and stainless steel brackets. Another study has reported that Nickel-free brackets produced relatively low friction levels. ${ }^{18}$ The manufacturers claim that, the increased hardness of Nickel-free over stainless steel aids in reduction of friction and as the Nickel-free brackets are cast and not milled or machined, the result is a smoother finish of the slot. One reason why no difference was observed in the friction levels between as-received 17-4 stainless steel and Nickel-free brackets was , may be that the slot dimensions of the Nickel-free brackets that were used were significantly less than that of the 17-4 stainless steel brackets thus increasing the friction values. The present study found that there was no significant difference in the amount of friction between the asreceived and recycled brackets for 17-4 stainless steel but the amount of friction in case of Nickel-free brackets increased significantly. This may be because with sandblasting the amount of surface roughness would have increased but the slot dimension had remained constant.

In this study, there was no significant difference in shear bond strength of as received 17-4 stainless steel and Nickel-free brackets. It has also been reported that higher bond strength was present in case of 17-4 stainless steel brackets as compared to Nickel- free brackets. ${ }^{9}$

In case of recycled brackets both 17-4 stainless steel and Nickel-free brackets had significantly higher bond strength as compared to as-received brackets. This increase in bond strength may be due to formation of additional resin tags between mesh base and enamel. Also it was suggested that sandblasting enhances retentive nature of metals by increasing the surface area and thinning the oxide layer and it may improve the bond at the bracket base.

\section{Conclusion}

The shear bond strength of recycled brackets was greater than as-received brackets for both 17-4 stainless steel and Nickel-free brackets. The slot dimensions of as-received brackets showed a lot of variation between the manufacturers but exhibited a wider dimension at face than at the base for both the manufacturers. On recycling the brackets manufactured by 17-4 stainless steel alloy showed a greater distortion in slot dimension as compared to Nickel-free alloy. The friction values for as-received brackets manufactured with 17-4 stainless steel and Nickel-free alloy were similar. On recycling the brackets manufactured by Nickel-free alloy showed a statistically significant increase in frictional values as compared to 17-4 stainless steel alloy.

\section{References}

1. Mui B, Rossouw PE, Kulkarni GV. Optimization of a procedure for rebonding dislodged orthodontic brackets. Angle Orthod 1999;69(3):276-81.

2. Burrow SJ. Friction and resistance to sliding in orthodontics: a critical review. Am J Orthod Dentofacial Orthop 2009;135(4):442-7.

3. Bandeira A, Santos MP, Pulitini G. Influence of thermal or chemical degradation on the frictional force of an experimental coated Niti wire. Angle Orthod 2011;81(3):484-9.

4. Kusy RP, Whitley JQ. Assessment of second order clearances between orthodontic archives and bracket slot via the critical contact angle for binding. Angle Orthod 1999;69:71-80.

5. Millet D, Mc Cabe JF, Gordon PH. The role of sandblasting on the retention of the metallic brackets applied with glass ionomer cement. Br J Orthod 1993;20:117-22.

6. Zinelis S, Annousaki O, Makou M, Eliades T. Metallurgical characterization of orthodontic brackets produced by metal injection moulding. Angle Orthod 2005;75:1024-31.

7. Retief DH, Denys FR. Finishing of enamel surfaces after debonding of orthodontic attachments. Angle Orthod 1979;49(1):1-10.

8. Eminkahyagil N, Arman A, Cetinsahin A, Karabulut E. Effect of resin removal methods on enamel and shear bond strength of rebonded brackets. Angle Orthod 2006;76:314-21.

9. Matasa CG. Pros and cons of the reuse of direct bonded appliances. Am J Orthod Dentofacial Orthop 1989;96:726. 
10. Cash AC, Good SA, Curtis RV, McDonald F. An evaluation of slot size in orthodontic brackets: are standards as expected? Angle Orthod 2004;74:450-53.

11. Kusy RP, Whitley JQ. Frictional resistances of metallined ceramic brackets versus conventional stainless steel brackets and development of 3-D friction maps. Angle Orthod 2001;71:364-74.

12. Buyukyilmaz T, Usumez S, Karaman AI. Effect of self etching primers on bond strength- are they reliable? Angle Orthod 2003;73:64-70.

13. Zinelis S, Annouski O, Eliades T, Makou M. Elemental composition of brazing alloys in metallic orthodontic brackets. Angle Orthod 2004;74:394-9.

14. Tangri K, Kumar P, Sharma P, Kumar K, Bagga DK, Sharma R. A comparison of the accuracy of 0.022 slots at Face, Base and Mesial and Distal surface of brackets marketed by different manufacturers. J Ind Orthod Soc 2012;46(3):132-6.

15. Choi SH, Kang DY, Hwang CJ. Surface roughness of three types of modern plastic bracket slot floors and frictional resistance. Angle Orthod 2014;84(1):177-83.

16. Pragati, Mehrotra P, Kapoor S, Dattada H, Rastogi N. Comparison of shear bond strength of new and recycled brackets with different mesh designs. J Ind Orthod Soc 2009;43(2):52-8.

17. Brown $\mathrm{P}$, Wagner W, Choi H. Orthodontic bracket slot dimensions as measured from entire bracket series. Angle Orthod 2015;85:678-82.

18. Lombardo L, Arreghini A, Bratti E, Mollica F, Spedicato G, Merlin M, Fortini A, Siciliani G. Comparative analysis of real and ideal wire-slot play in square and rectangular archwires. Angle Orthod 2015;85:848-58. 